\title{
COMBINED EFFECTS OF VISCOUS DISSIPATION AND HEAT GENERATION ON NATURAL CONVECTION FLOW ALONG A VERTICAL WAVY SURFACE
}

\author{
K. H. Kabir ${ }^{1}$, M. A. Alim ${ }^{2 *}$, L. S. Andallah ${ }^{3}$ and Saika Mahjabin ${ }^{4}$ \\ ${ }^{1}$ Department of Mathematics, Mohammadpur Kendriya College, Dhaka, Bangladesh. \\ ${ }^{2}$ Department of Mathematics, Bangladesh University of Engineering and Technology, Dhaka, Bangladesh. \\ ${ }^{3}$ Department of Mathematics, Jahangirnagar University, Savar, Bangladesh. \\ ${ }^{4}$ Department of Mathematics, National University, Gazipur 1704. \\ "Corresponding e-mail: maalim@math.buet.ac.bd
}

\begin{abstract}
In this paper, the effects of viscous dissipation on natural convection flow along a uniformly heated vertical wavy surface with heat generation have been investigated. The governing boundary layer equations are first transformed into a non-dimensional form using suitable set of dimensionless variables. The resulting nonlinear systems of partial differential equations are mapped into the domain of a vertical flat plate and then solved numerically employing the Keller-box method. The numerical results of the surface shear stress in terms of skin friction coefficient and the rate of heat transfer in terms of local Nusselt number, the velocity as well as the temperature profiles are shown graphically and in tabular form for different values of physical parameters namely, viscous dissipation parameter Vd, heat generation parameter $Q$ and Prandtl number Pr.
\end{abstract}

Keywords: Natural convection, uniform surface temperature, wavy surface, viscous dissipation, Prandtl number, heat generation.

\section{INTRODUCTION}

Natural convection boundary layer flow along various geometrical shapes such as vertical flat plate, cylinder, sphere, vertical wavy surface etc, have been studied by many investigators and it has been a very popular research topic for many years. The viscous dissipation effect plays an important role in natural convection in various devices which are subjected to large deceleration or which operate at high rotational speeds and also in strong gravitational field processes on large scales (on large planets) and in geological processes. The natural convection from an isothermal vertical wavy surface was first studied by $\mathrm{Yao}^{1}$ and using an extended Prantdl's transposition theorem and a finite-difference scheme. He proposed a simple transformation to study the natural convection heat transfer from isothermal vertical wavy surfaces, such as sinusoidal surface. Moulic and $\mathrm{Yao}^{2}$ also investigated mixed convection heat transfer along a vertical wavy surface. Combined effects of thermal and mass diffusion on the natural convection flow of a viscous incompressible fluid along a vertical wavy surface have been investigated by Hossain and Rees ${ }^{3}$. Wang and $\mathrm{Chen}^{4}$ investigated transient force and free convection along a vertical wavy surface in micropolar fluid. Natural and mixed convection heat and mass transfer along a vertical wavy surface have been investigated by Jang et al. ${ }^{5}$ and Jang and Yan ${ }^{6}$. The influence and importance of viscous stress work effects in laminar flows have been examined by Gebhart ${ }^{7}$ and Gebhart and Mollendorf ${ }^{8}$. In both of the investigations special flows over semi-infinite flat surfaces parallel to the direction of body force were considered. Gebhart ${ }^{7}$ considered flows generated by the plate surface temperatures and Gebhart and Mollendorf ${ }^{8}$ considered flows generated by plate surface temperatures. Zakerullah $^{9}$ has been investigated the viscous dissipation and pressure work effects in axisymmetric natural convection flows. In the present study, the effect of viscous dissipation on natural convection flow of viscous incompressible fluid along a uniformly heated vertical wavy surface has been investigated. The governing partial differential equations are reduced to locally non-similar partial differential forms by adopting some appropriate transformations. The transformed boundary layer equations are solved numerically using finite difference method known as Keller box technique ${ }^{10}$ described by Keller and later by Cebeci and Bradshaw ${ }^{11}$ along with Newton's linearization approximation. Numerical results have been obtained in terms of local skin friction coefficient and the rate of heat transfer in terms of local Nusselt number, the velocity as well as the tempreture profiles for a selection of relevant physical parameters are shown graphically and in tabular form.

\section{Formulation of the Problem}

Steady two dimensional laminar free convection boundary layer flow of a viscous incompressible and electrically conducting fluid along a vertical wavy surface in presence of uniform transverse magnetic field is considered. It is assumed that the wavy surface is electrically insulated and is maintained at a uniform temperature $T_{w}$. The surface temperature $T_{w}$ is greater 
than the ambient fluid temperature $T_{\infty}$ that is, $T_{w}>T_{\infty}$. A uniform magnetic field of strength $\beta_{0}$ is imposed along the $Y$-axis i.e. normal direction to the surface and $X$ -axis is taken along the surface. The flow configuration of the wavy surface and the two-dimensional cartesian coordinate system are shown in Fig. 1.

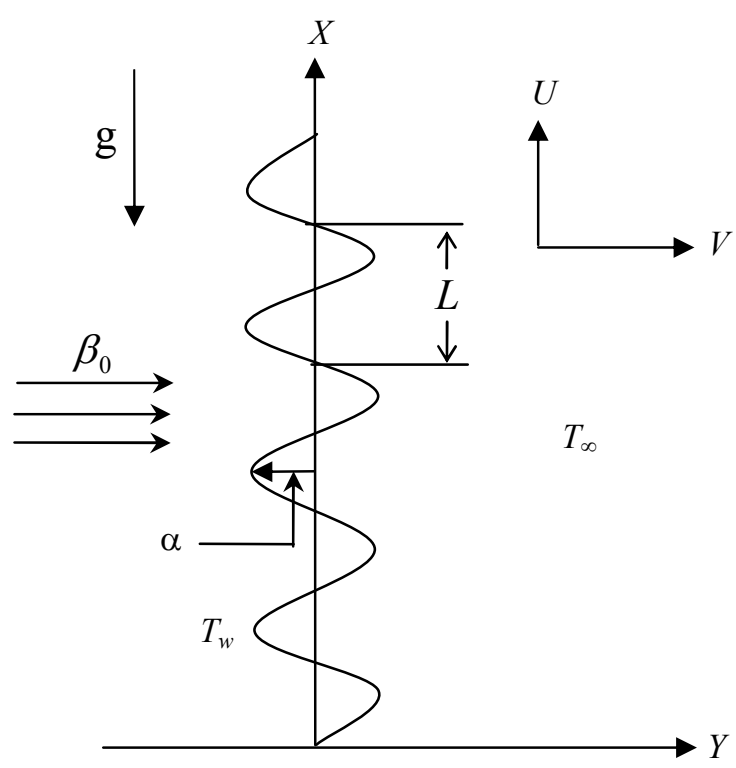

Figure 1. Physical model and coordinate system.

The boundary layer analysis outlined below allows $\bar{\sigma}(X)$ being arbitrary, but our detailed numerical work assumed that the surface exhibits sinusoidal deformations. The wavy surface may be defined by

$Y_{w}=\bar{\sigma}(X)=\alpha \sin \left(\frac{n \pi X}{L}\right)$

where $\alpha$ is the amplitude and $L$ is the wave length associated with the wavy surface.

The governing equations of such flow of magnetic field in presence of heat generation with viscosity variation along a vertical wavy surface under the usual Boussinesq approximations can be written in a dimensional form as:

Continuity Equation

$\frac{\partial U}{\partial X}+\frac{\partial V}{\partial Y}=0$

$X$-Momentum Equation

$$
\begin{aligned}
U \frac{\partial U}{\partial X} & +V \frac{\partial U}{\partial Y}=-\frac{1}{\rho} \frac{\partial P}{\partial X} \\
& +\frac{1}{\rho} \nabla \cdot(\mu \nabla U)+g \beta\left(T-T_{\infty}\right)
\end{aligned}
$$

$Y$-Momentum Equation

$U \frac{\partial V}{\partial X}+V \frac{\partial V}{\partial Y}=-\frac{1}{\rho} \frac{\partial P}{\partial Y}+\frac{1}{\rho} \nabla \cdot(\mu \nabla V)$

Energy Equation

$$
\begin{aligned}
U \frac{\partial T}{\partial X} & +V \frac{\partial T}{\partial Y}=\frac{k}{\rho C_{P}} \nabla^{2} T \\
& +\frac{Q_{o}}{\rho C_{p}}\left(T-T_{\infty}\right)+\frac{v}{\rho C_{P}}\left(\frac{\partial U}{\partial Y}\right)^{2}
\end{aligned}
$$

where $(X, Y)$ are the dimensional coordinates along and normal to the tangent of the surface and $(U, V)$ are the velocity components parallel to $(X, Y), g$ is the acceleration due to earth gravity, $P$ is the dimensional pressure of the fluid, $T$ is the temperature of the fluid in the boundary layer, $C_{P}$ is the specific heat at constant pressure, $\mu$ is the dynamic viscosity of the fluid in the boundary layer region depending on the fluid temperature, $\rho$ is the density, $v$ is the kinematic viscosity, where $v=\mu / \rho, k$ is the thermal conductivity of the fluid, $\beta$ is the volumetric coefficient of thermal expansion, $\beta_{0}$ is the strength of magnetic field, $\sigma_{0}$ is the electrical conductivity of the fluid, $Q_{0}$ is the heat generation constant and $\nabla^{2}$ is the Laplacian operator, where $\nabla^{2}=\frac{\partial^{2}}{\partial x^{2}}+\frac{\partial^{2}}{\partial y^{2}}$.

The boundary conditions for the present problem are

$U=0, V=0, T=T_{w}$ at $Y=Y_{w}=\bar{\sigma}(X)$

$U=0, T=T_{\infty}, P=p_{\infty}$ as $Y \rightarrow \infty$

Using Prandtl's transposition theorem to transform the irregular wavy surface into a flat surface as extended by Yao [1] and boundary layer approximation, the following dimensionless variables are introduced for non-dimensionalizing the governing equations

$$
\begin{aligned}
& x=\frac{X}{L}, y=\frac{Y-\bar{\sigma}}{L} G r^{\frac{1}{4}}, p=\frac{L^{2}}{\rho v^{2}} G r^{-1} P \\
& u=\frac{\rho L}{\mu_{\infty}} G r^{-1 / 2} U, v=\frac{\rho L}{\mu_{\infty}} G r^{-1 / 4}\left(V-\sigma_{x} U\right), \\
& \theta=\frac{T-T_{\infty}}{T_{w}-T_{\infty}} \\
& \sigma_{x}=\frac{d \bar{\sigma}}{d X}=\frac{d \sigma}{d x}, G r=\frac{g \beta\left(T_{w}-T_{\infty}\right)}{v^{2}} L^{3}
\end{aligned}
$$

where $\theta$ is the dimensionless temperature function and $(u, v)$ are the dimensionless velocity components parallel to $(x, y)$ and $G r$ is the Grashof number. Now introducing the dimensionless dependent and independent variables into equations (2)-(5), the 
following dimensionless form of the governing equations are obtained after ignoring terms of smaller orders of magnitude in the Grashof number $G r$.

$$
\begin{aligned}
& \frac{\partial u}{\partial x}+\frac{\partial v}{\partial y}=0 \\
& u \frac{\partial u}{\partial x}+v \frac{\partial u}{\partial y}=-\frac{\partial p}{\partial x}+G r^{1 / 4} \sigma_{x} \frac{\partial p}{\partial y} \\
& +\left(1+\sigma_{x}^{2}\right) \frac{\partial^{2} u}{\partial y^{2}}+\theta \\
& \sigma_{x}\left(u \frac{\partial u}{\partial x}+v \frac{\partial u}{\partial y}\right)=-G r^{1 / 4} \frac{\partial p}{\partial y} \\
& +\sigma_{x}\left(1+\sigma_{x}^{2}\right) \frac{\partial^{2} u}{\partial y^{2}}-\sigma_{x x} u^{2} \\
& u \frac{\partial \theta}{\partial x}+v \frac{\partial \theta}{\partial y}=\frac{1}{\operatorname{Pr}}\left(1+\sigma_{x}^{2}\right) \frac{\partial^{2} \theta}{\partial y^{2}} \\
& +Q \theta+V d\left(\frac{\partial u}{\partial y}\right)^{2}
\end{aligned}
$$

It is worth noting that the $\sigma_{x}$ and $\sigma_{x x}$ indicate the first and second derivetives of $\sigma$ with respect to $x$, therefore, $\sigma_{x}=$ $d \bar{\sigma} / d X=d \sigma / d x$ and $\sigma_{x x}=d \sigma_{x} / d x$.

In the above equations $\operatorname{Pr}, Q$ and $V d$ are respectively known as the Prandtl number, heat generation parameter and viscous dissipation parameter, which are defined as

$$
\begin{aligned}
& \operatorname{Pr}=\frac{C_{p} \mu_{\infty}}{k}, \quad Q=\frac{Q_{o} L^{2}}{\mu C_{p} G r^{\frac{1}{2}}} \text { and } \\
& V d=\frac{G r}{L^{2} C_{p}\left(T_{w}-T_{\infty}\right)}
\end{aligned}
$$

For the present problem the pressure gradient $(\partial p / \partial x=0)$ is zero. Thus, the elimination of $\partial p / \partial y$ from equations (9) and (10) leads to

$$
\begin{aligned}
& u \frac{\partial u}{\partial x}+v \frac{\partial u}{\partial y}=\left(1+\sigma_{x}^{2}\right) \frac{\partial^{2} u}{\partial y^{2}} \\
& -\frac{\sigma_{x} \sigma_{x x}}{1+\sigma_{x}^{2}} u^{2}+\frac{1}{1+\sigma_{x}^{2}} \theta
\end{aligned}
$$

The corresponding boundary conditions for the present problem then turn into

$$
\left.\begin{array}{l}
u=v=0, \theta=1 \text { at } y=0 \\
u=\theta=0, \text { as } y \rightarrow \infty
\end{array}\right\}
$$

Now we introduce the following transformations to reduce the governing equations to a convenient form:

$$
\begin{aligned}
& \psi=x^{3 / 4} f(x, \eta), \quad \eta=y x^{-1 / 4} \\
& \theta=\theta(x, \eta)
\end{aligned}
$$

where $f(\mathrm{x}, \eta)$ is the dimensionless stream function, $\eta$ is the dimensionless similarity variable and $\psi$ is the stream function that satisfies the continuity equation (8) and is related to the velocity components in the usual way as

$u=\frac{\partial \psi}{\partial y}, \quad v=-\frac{\partial \psi}{\partial x}$

Introducing the transformations given in equation (15) and using (16) into equations (13) and (11) are transformed into the new co-ordinate system. Thus the resulting equations are

$$
\begin{aligned}
& \left(1+\sigma_{x}^{2}\right) f^{\prime \prime \prime}+\frac{3}{4} f f^{\prime \prime}-\left(\frac{1}{2}+\frac{x \sigma_{x} \sigma_{x x}}{1+\sigma_{x}^{2}}\right) f^{\prime 2} \\
& +\frac{1}{1+\sigma_{x}^{2}} \theta=x\left(f^{\prime} \frac{\partial f^{\prime}}{\partial x}-f^{\prime \prime} \frac{\partial f}{\partial x}\right) \\
& \frac{1}{\operatorname{Pr}}\left(1+\sigma_{x}^{2}\right) \theta^{\prime \prime}+\frac{3}{4} f \theta^{\prime}+x^{1 / 2} Q \theta \\
& +V d x\left(f^{\prime \prime}\right)^{2}=x\left(f^{\prime} \frac{\partial \theta}{\partial x}-\theta^{\prime} \frac{\partial f}{\partial x}\right)
\end{aligned}
$$

The boundary conditions (14) now take the following form:

$$
\left.\begin{array}{l}
f(x, o)=f^{\prime}(x, o)=0, \quad \theta(x, o)=1 \\
f^{\prime}(x, \infty)=0, \quad \theta(x, \infty)=0
\end{array}\right\}
$$

Here prime denote the differentiation with respect to $\eta$.

However, once we know the values of the functions $f$ and $\theta$ and their derivatives, it is important to calculate the values of the rate of heat transfer in terms of local Nusselt number $N u_{x}$ and the shearing stress $\tau_{\mathrm{w}}$ in terms of the local skin friction coefficient $C_{f x}$ from the following relations:

$N u_{x}=\frac{q_{w} X}{k\left(T_{w}-T_{\infty}\right)} \quad$ and $\quad C_{f x}=\frac{2 \tau_{w}}{\rho U^{2}}$

where, $q_{w}=-k(\bar{n} \cdot \nabla T)_{y=0}$

and $\tau_{w}=\left(\begin{array}{ll}\mu & \bar{n} . \nabla \bar{u}\end{array}\right)_{y=0}$

Also $U=\mu_{\infty} G r^{1 / 2} / \rho L$

Here $\bar{n}=\frac{\bar{i} f_{x}+\bar{j} f_{y}}{\sqrt{f_{x}^{2}+f_{y}^{2}}}$ is the unit normal to the surface. Using the transformation (15) and (21) into equation (20) the rate of heat transfer in terms of the 
local Nusselt number $N u_{x}$ and the local skin friction coefficient $C_{f x}$ take the following forms:

$$
\begin{aligned}
& N u_{x} G r^{-1 / 4} x^{-3 / 4}=-\sqrt{1+\sigma_{x}^{2}} \theta^{\prime}(x, o) \\
& C_{f x}(G r / x)^{1 / 4} / 2=\sqrt{1+\sigma_{x}^{2}} \quad f^{\prime \prime}(x, o)
\end{aligned}
$$

\section{RESULTS AND DISCUSSIONS}

The effects of viscous dissipation on natural convection flow of viscous incompressible fluid along a uniformly heated vertical wavy surface have been investigated. Although there are four parameters of interest in the present problem, the effects of viscous dissipation $V d$, the heat generation parameter $\mathrm{Q}$, Prandtl number $\operatorname{Pr}$ and the amplitude of the wavy surface $\alpha$ on the surface shear stress in terms of local skin friction coefficient, the rate of heat transfer in terms of the local Nusselt number, the velocity and temperature profiles, the streamlines and the isotherms. Numerical values of local shearing stress and the rate of heat transfer are calculated from equations (22) and (23) in terms of the skin-friction coefficients $C_{f x}$ and Nusselt number $N u_{x}$ respectively for a wide range of the axial distance variable $x$ starting from the leading edge for different values of the parameters $\operatorname{Pr}, Q, V d$ and $\alpha$.
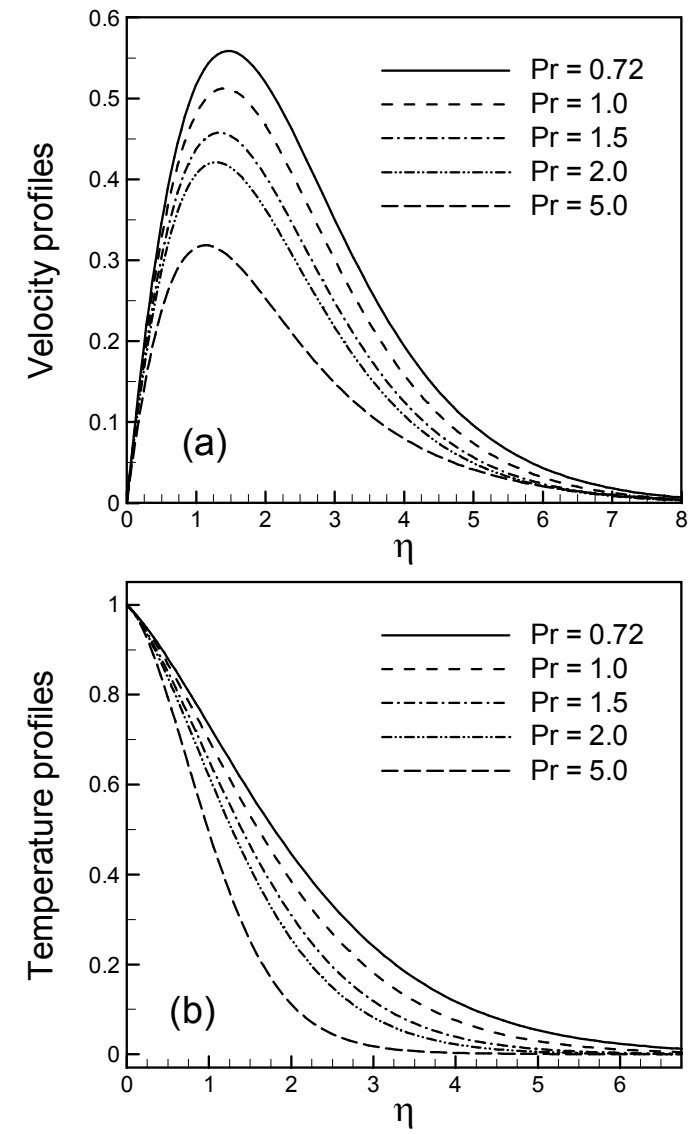

Figure 2. (a) Velocity and (b) Temperature profiles against $\eta$ for different values of $\operatorname{Pr}$ with $\alpha=0.1$, $\mathrm{Q}=0.5$ and $V d=10$.
Solutions are obtained in terms of velocity profiles, temperature profiles against $\eta$ and the skin friction coefficients $C_{f x}$, the rate of heat transfer in terms of the Nusselt number $N u_{x}$ at any position of $x$ presented graphically for selected values of Prandtl number $\operatorname{Pr}=0.72,1.0,1.5,2.05 .0$, heat generation parameter $Q=0.0,0.5,1.0,1.5,2.0$ and viscous dissipation parameter $V d=0.0,5.0,10.0,15.0,20.0$. The different values of Prandtl number $\operatorname{Pr}$ considered while $\alpha=0.1$. $\mathrm{Q}=0.5$, and $V d=10.0$. The different values of heat generation parameter $Q$ considered while $\alpha=0.1$. $P r=$ 0.72 and $V d=25.0$. The different values of viscous dissipation parameter $V d$ considered while $\alpha=0.1 . P r$ $=0.72$, and $Q=0.5$ are also observed and these are shown graphically in Figs 2 - 7.
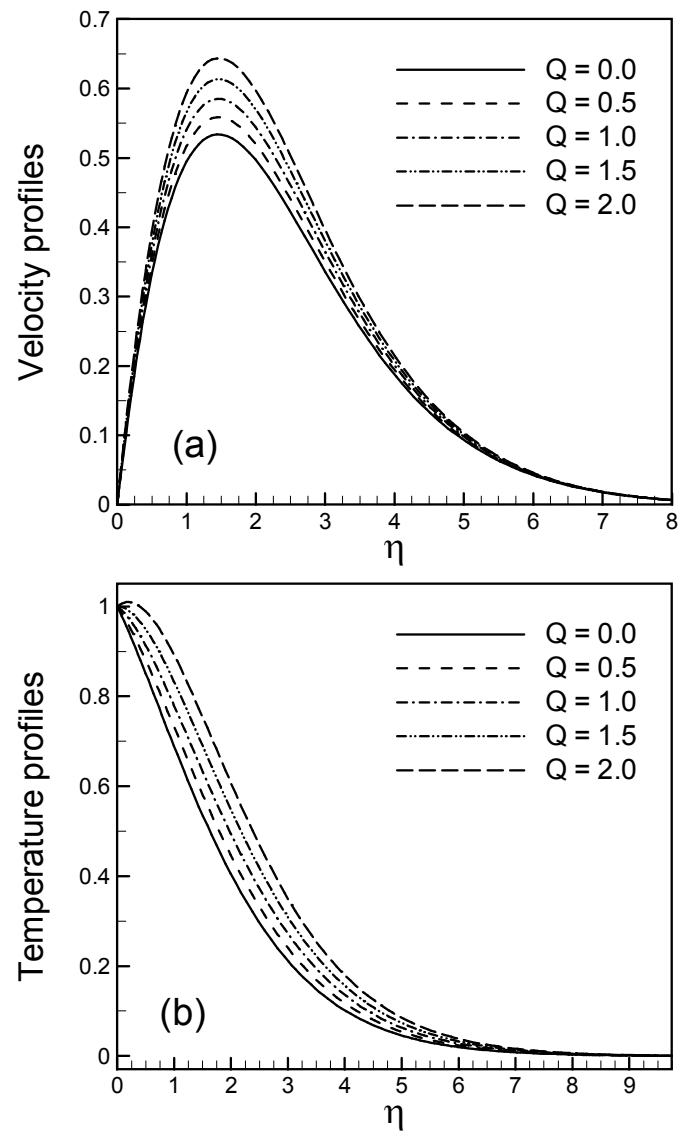

Figure 3. (a) Velocity and (b) Temperature profiles against $\eta$ for different values of $Q$ with $\operatorname{Pr}=0.72, \alpha=0.1$ and $\mathrm{Vd}=10$.

In Fig. 2(a) and 2(b) the effects of Prandtl number $\operatorname{Pr}$ on velocity and temperature for $\alpha=0.1 . Q=0.5$, and $V d=10.0$ have been shown. The different values of Prandtl number have been chosen as $P r=0.72,1.0$, 1.5, 2.0, 5.0. For the higher values of Prandtl number $\operatorname{Pr}$ both the velocity and the temperature decreases such that there exists a local maximum of the velocity within the boundary layer. The maximum values of velocities are recorded as $0.53860,0.49030,0.43351$, 0.39544 and 0.28892 for Prandtl number $\operatorname{Pr}=0.72$, 

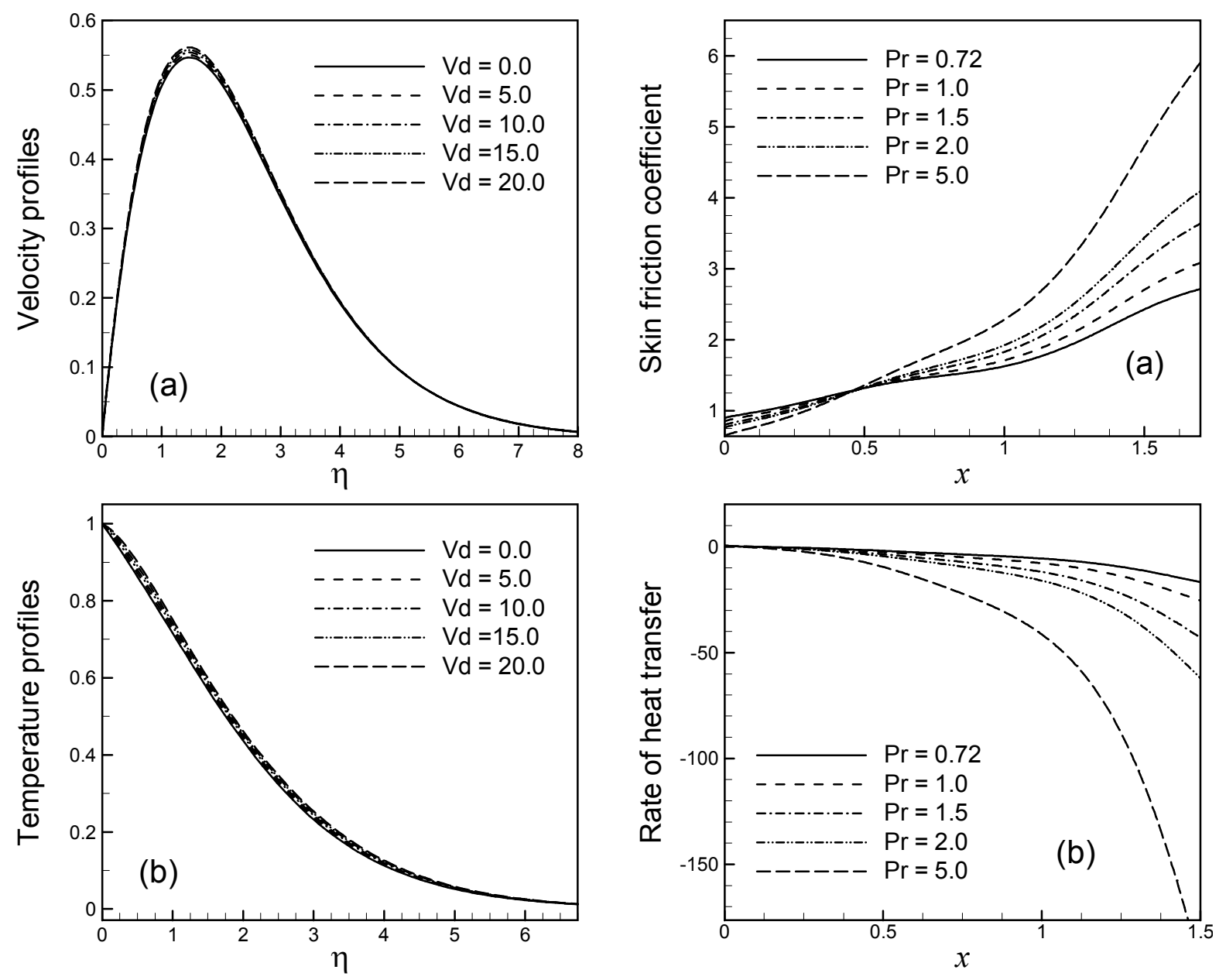

Figure 4.: (a) Velocity and (b) Temperature profiles against $\eta$ for different values of $\mathrm{Vd}$ with $\operatorname{Pr}=0.72$,

$$
\alpha=0.1 \text { and } Q=0.5 \text {. }
$$

Figure 5. (a) Skin friction coefficient and (b) Rate of heat transfer against $\mathrm{x}$ for different values of $\mathrm{Pr}$ with $\alpha=0.1, \mathrm{Vd}=10.0$ and $\mathrm{Q}=0.5$.

$1.0,1.5,2.0$ and 5.0 at the position of $\eta=1.36929$, $1.30254,1.23788,1.23788$ and 1.05539 respectively and the maximum velocity decreases by $46.36 \%$. The velocities are recorded as $0.53503,0.48911,0.43351$, 0.39544 and 0.28582 for Prandtl number $P r=0.72$, $1.0,1.5,2.0$ and 5.0 at the particular position of $\eta=1.23788$ and the velocity decreases by $46.68 \%$. The velocities fall down slowly have been seen from Fig. 2(a). The values of temperature are recorded as $0.50125,0.44706,0.37840,0.32953$ and 0.18365 for Prandtl number $\operatorname{Pr}=0.72,1.0,1.5,2.0$ and 5.0 at the position of $\eta=1.50946$ and the temperature decreases by $63.36 \%$. Figure 2(b) displays the results that the change of temperature profiles in the $\eta$-direction reveals the typical temperature profiles for natural convection boundary layer flow, i.e., the temperature is zero at the boundary wall. It is observed that the velocity as well as the boundary layer thickness decreases and the temperature as well as the thermal boundary layer thickness decreases for the increasing values of Prandtl number. The effects for different values of the heat generation parameter $Q$ on the velocity and temperature profiles with $\alpha=0.1 . P r=$ $0.72, V d=10.0$ have been presented graphically in Figs 3(a) and 3(b) respectively. For the higher values of the heat generation parameter $Q(=0.0,0.5,1.0,1.5$, $2.0)$ both the velocity and the temperature increases. In Figs 4(a) and 4(b) the effects for different values of the viscous dissipation parameter $V d$ on the velocity and temperature profiles with $\alpha=0.1 . \operatorname{Pr}=0.72, Q=0.5$ have been shown graphically..It has been seen from Fig. 4(a) that as the viscous dissipation parameter $V d$ increases, the velocities rising up to the position of $\eta=1.43822$ for viscous dissipation parameter $V d=0.0,5.0,10.0,15.0,20.0$ and from that position of $\eta$ velocities fall down slowly and finally approaches to zero. It is also observed from Fig. 4(b) that as the viscous dissipation parameter $V d$ increases, the temperature profiles increases. The maximum values of velocities are recorded as $0.52232,0.52570,0.52909$, 0.53251 and 0.53595 for viscous dissipation parameter $V d=0.0,5.0,10.0,15.0,20.0$ respectively which occur at the same position $\eta=1.43822$ and the maximum velocity increases by $2.61 \%$. Temperatures are recorded as $0.17726,0.18163,0.18604,0.19050$ and 
0.19501 for viscous dissipation parameter $V d=0.0$, $5.0,10.0,15.0,20.0$ respectively at the same position of $\eta=3.20$ and the temperature profiles increases by $10.01 \%$. Both the velocity and temperature profiles accumulate nearly in the following points where $\eta=7.55722$ and $\eta=7.86828$ respectively for viscous dissipation parameter $V d=0.0,5.0,10.0,15.0,20.0$ That is, velocity boundary layer thickness and thermal boundary layer thickness are unchanged.
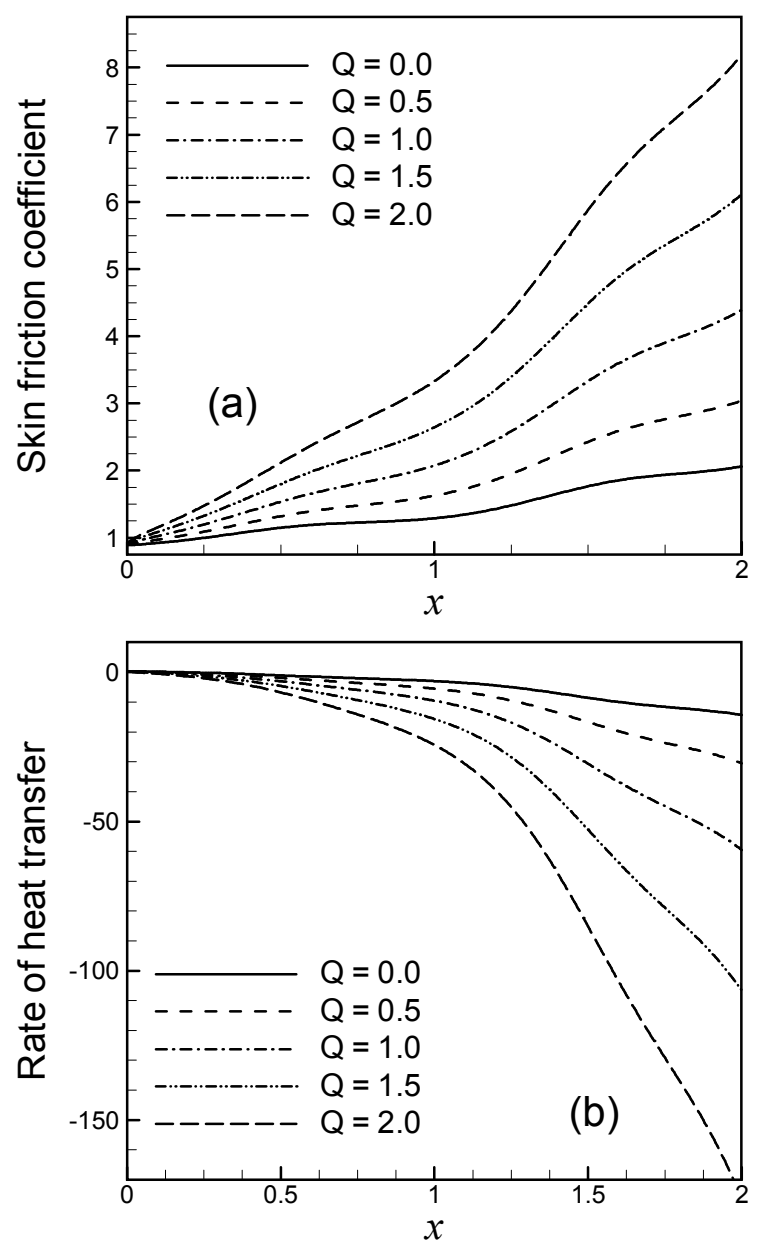

Figure 6. (a) Skin friction coefficient and (b) Rate of heat transfer against $x$ for different values of $Q$ with $\operatorname{Pr}=0.72, \alpha=0.1$ and $\mathrm{Vd}=10$.

In Figs 5(a) and 5 (b) the skin friction coefficient $C_{f x}$ and local rate of heat transfer $N u_{x}$ for different values of Prandtl number $P r$ with $\alpha=0.10$. Q $=0.5$, and $V d=10.0$ have been displayed. It is observed from the Fig. 5(a) that for higher values of Prandtl number skin friction decreasing upto the axial position of $\mathrm{x}=1.0$ and then skin friction becomes constant for all values of $\mathrm{Pr}$ that is, skin friction coefficient meet together at the position of $\mathrm{x}=1.0$ and cross the sides that means after the axial position of $\mathrm{x}=1.0$ skin friction is increasing with Prandtl number but frictional force at the wall always rising towards downstream. It is seen from the Fig. 5(b) that for higher values of Prandtl number the
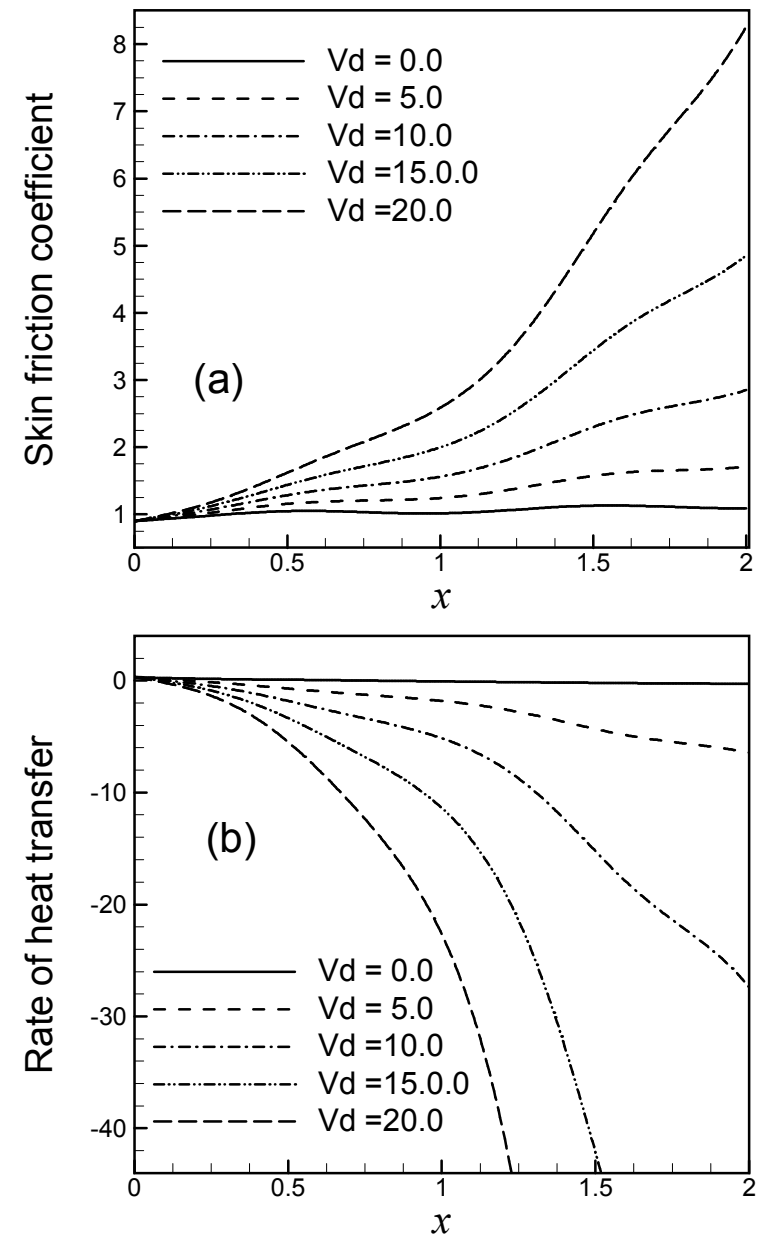

Figure 7. (a) Skin friction coefficient and (b) Rate of heat transfer against $\mathrm{x}$ for different values of $\mathrm{Vd}$ with $\operatorname{Pr}=0.72, \alpha=0.1$ and $Q=0.5$.

rate of heat transfer decreases that is heat transfer slows down for higher Prandtl number. In Figs 6(a) and 6 (b) effects of the heat generation parameter $Q$ on skin friction and the rate of heat transfer with $\alpha=0.1$. $P r=$ 0.72 , and $V d=10.0$ have been presented grafically. From Fig. 6(a) it is found that skin friction increases significantly for the greater values of the heat generation parameter $Q$. However rate of heat transfer opposite pattern due to the higher values of the heat generation parameter $Q$ which are presented in Fig. $6(\mathrm{~b})$. The different values of viscous dissipation parameter $V d$ with $\alpha=0.1 . P r=0.72$, and $Q=0.5$ of the skin friction coefficients and the rate of heat transfer are shown graphically in Figs 7(a) and 7 (b) respectively. In this case the values of local skin friction coefficient $C_{f x}$ are recorded to be $0.91799,1.19362,1.65790,2.41420$ and 3.60650 for $V d=0.0,5.0,10 ., 15.0,20.0$ which occur at same point $x=1.51$. From the Fig. 7(a), it is observed that at $x=1.51$, the skin friction coefficient increases by $292.87 \%$ due to the higher value of viscous dissipation parameter $V d$. However, the values of rate of heat transfer are found to be 0.34382 , $-1.91932,-7.63071,-21.73558$, and -55.25804 for $V d$ 
$=0.0,5.0,10.0,15.0,20.0$ which occur at same point $x$ $=1.51$. The rate of heat transfer coefficient decreases by $16171.79 \%$ due to the increased value of viscous dissipation parameter $V d=0.0,5.0,10.0,15.0,20.0$. It is seen from the Fig. 7(b) that for higher values of viscous dissipation parameter the rate of heat transfer decreases that is heat transfer slows down for higher viscous dissipation parameter $V d$.

\section{CONCLUSIONS}

The effects of the Prandtl number $P r$, the heat generation parameter $Q$, the viscous dissipation parameter $V d$ and the amplitude of wavy surface $\alpha$ on natural convection flow of viscous incompressible fluid along a uniformly heated vertical wavy surface have been studied. From the present investigations the following conclusions may be drawn:

For the higher values of Prandtl number $\mathrm{Pr}$ the velocity profiles, the temperature profiles decreases slowly and finally approach to zero, the rate of heat transfer $N u_{x}$, decreases while the skin friction coefficient $C_{f x}$ initialy decreases and at the position of $\mathrm{x}=1.0$ becomes constant that is skin friction meet at the point and then cross the side and increasing with Prandtl number $\mathrm{Pr}$.

Increasing values of the heat generation parameter $Q$ the velocity, the temperature and the skin friction coefficient $C_{f x}$ increases. The rate of heat transfer coefficient $N u_{x}$ decreases due to the increased value of the heat generation parameter $Q$.

Improved value of the viscous dissipation parameter $V d$ the velocity profiles, the temperature profiles and the local skin friction coefficient $C_{f x_{x}}$ increases. The rate of heat transfer coefficient $N u_{x}$ decreases due to the increased value of viscous dissipation. strength.

\section{NOMENCLATURE}

$C_{f x} \quad$ Local skin friction coefficient

$C_{p} \quad$ Specific heat at constant pressure $\left[\mathrm{J} \cdot \mathrm{kg}^{-1} \cdot \mathrm{K}^{-1}\right]$

$f \quad$ Dimensionless stream function

$g \quad$ Acceleration due to gravity $\left[\mathrm{ms}^{-2}\right]$

Gr Grashof number

$k \quad$ Thermal conductivity $\left[\mathrm{Wm}^{-1} \mathrm{~K}^{-1}\right]$

$k_{\infty} \quad$ Thermal conductivity of the ambient fluid $\left[\mathrm{Wm}^{-1} \mathrm{~K}^{-1}\right]$

$L \quad$ Characteristic length associated with the wavy surface $[\mathrm{m}]$

$\bar{n} \quad$ Unit normal to the surface

$N u_{x} \quad$ Local Nusselt number

$P \quad$ Pressure of the fluid $\left[\mathrm{Nm}^{-2}\right]$

Pr Prandtl number

$Q \quad$ Heat generation parameter
$Q_{0} \quad$ Heat generation constant
$q_{w} \quad$ Heat flux at the surface $\left[\mathrm{Wm}^{-2}\right]$
$T$ Temperature of the fluid in the boundary layer [K]
$T_{w} \quad$ Temperature at the surface $[\mathrm{K}]$
$T_{\infty} \quad$ Temperature of the ambient fluid [K]
$u, v$ Dimensionless velocity components along the $(x, y)$ axes $\left[\mathrm{ms}^{-1}\right]$

$x, y \quad$ Axis in the direction along and normal to the tangent of the surface

\section{GREEK SYMBOLS}

$\alpha \quad$ Amplitude of the surface waves

$\beta \quad$ Volumetric coefficient of thermal expansion $\left[\mathrm{K}^{-1}\right]$

$\eta \quad$ Dimensionless similarity variable

$\theta$ Dimensionless temperature function

$\psi \quad$ Stream function $\left[\mathrm{m}^{2} \mathrm{~s}^{-1}\right]$

$\mu \quad$ Viscosity of the fluid $\left[\mathrm{kgm}^{-1} \mathrm{~s}^{-1}\right]$

$\mu_{\infty} \quad$ Viscosity of the ambient fluid

$v \quad$ Kinematic viscosity $\left[\mathrm{m}^{2} \mathrm{~s}^{-1}\right]$

$\rho \quad$ Density of the fluid $\left[\mathrm{kgm}^{-3}\right]$

$\sigma_{0} \quad$ Electrical conductivity

$\tau_{w} \quad$ Shearing stress

\section{REFERENCES}

1. L. S. Yao, 1983, Natural Convection along a Vertical Wavy Surface, ASME Journal of Heat Transfer, Vol. 105, No.3, pp. $465-468$.

2. S.G. Moulic, L.S. Yao, 1989, Mixed Convection along Wavy Surface, ASME Journal of Heat Transfer, Vol. 111, No.4, pp. 974-979.

3. M.A. Hossain, D.A.S. Rees, 1999, Combined Heat and Mass Transfer in Natural Convection Flow from a Vertical Wavy Surface, Acta Mechanica, Vol. 136, No.3, pp. 133-141.

4. C. C. Wang, C. K. Chen, 2001, Transient Force and Free Convection along a Vertical Wavy Surface in Micropolar Fluid, International Journal of Heat and Mass Transfer, Vol. 44, No.17, pp. 3241-3251.

5. J. H. Jang, W. M. Yan, H. C. Liu, 2003, Natural Convection Heat and Mass Transfer along a Vertical Wavy Surface, International Journal of Heat and Mass Transfer, Vol. 46, No.6, pp. 1075-1083.

6. J. H. Jang, W. M. Yan, 2004, Mixed Convection Heat and Mass Transfer along a Vertical Wavy Surface, International Journal of Heat and Mass Transfer, Vol. 47, No.3, pp. 419-428. 
7. Gebhart, B, 1962, Effects of Viscous Dissipation in Natural Convection, Journal of Fluid Mechanics, Vol. 14, No. 2, pp. 225-232.

8. Gebhart,B., Mollendorf, J, 1969, Viscous Dissipation in External Natural Convection Flows, Journal of Fluid Mechanics, Vol.38, No. 1, pp. 97-107. 9. Zakerullah, M, 1972,Viscous Dissipation and Pressure Work Effects in Axisymmetric Natural Convection Flows, Ganit(J. Bangladesh Math. Soc.) Vol. 2. No.1, pp. 43.
10. H. B. Keller, 1978, Numerical Methods in Boundary Layer Theory, Annual Review of Fluid Mechanics, Vol. 10, No.1, pp. 417-433.

11. T. Cebeci , P. Bradshaw, 1984, Physical and Computational Aspects of Convective Heat Transfer, Springer, New York. 